\title{
Symposium on stochastic volatility: an introductory overview
}

\author{
Frederi G. Viens
}

Published online: 16 June 2010

(C) Springer-Verlag 2010

\begin{abstract}
We describe the context and summarize the contents of the ten contributions to the Symposium on Stochastic Volatility. These articles concentrate mainly on questions pertaining to option pricing under various uncertainty assumptions about market volatility. Tools from stochastic analysis and statistical inference are used to present solutions via explicit computations or numerical methods, with model estimation and calibration based on market and simulated data.
\end{abstract}

Keywords Stochastic volatility - Option pricing - Calibration · Estimation . Stochastic $\cdot$ Stock market

JEL Classification $\mathrm{C} 1 \cdot \mathrm{C} 5 \cdot \mathrm{C} 6 \cdot \mathrm{G} 1$

The celebrated continuous-time asset price model of Black, Merton, and Scholes is as popular in investment finance today as ever, by virtue of its simplicity and versatility. The principal cost of this simplicity is the model's long-acknowledged inadequacy in describing the volatility of financial markets. Initially, it was assumed that the log returns of discounted asset and index prices are exactly proportional to Brownian increments, the proportionality constant $\sigma$ being the so-called volatility. Since the mid 1980's, quantitative finance specialists have proposed replacing $\sigma$ by a stochastic process in its own right, often denoted by $\sigma_{t}$, and generically called stochastic volatility (SV). Since there is no specific canonical model of SV that can account simultaneously for all the features of financial volatility observed in markets, the quantitative study of SV has been and continues to be vigorously studied — and sometimes hotly disputed.

F. G. Viens $(\varangle)$

Department of Statistics, Purdue University,

150 N. University St., West Lafayette, IN 47907-2067, USA

e-mail: viens@purdue.edu 
In this Symposium issue of the Annals of Finance, we look at some of the most recent advances in the quantitative study of SV, covering mathematical, statistical, econometric, and computational aspects, all with sound financial interpretation as the primary motivator. We will encounter a rich variety of topics and methods, whose common goal is to explain the complexity of swings in market data.

The main financial emphasis in this issue is on the pricing of options and other financial derivatives. An underlying unifying philosophy is that option prices contain the most complete information about volatility, since they include a usage of past and present asset price movements, and translate market makers' expectations of where the markets are headed. In accordance with this belief, the authors of this Symposium propose a number of methods in which it is possible to let option prices speak for themselves, by calibrating models against them.

Among the methods encountered here, some allow explicit option prices, a good path to reconcile financial intuition and precise quantitative statements. Other methods require Monte-Carlo techniques, for solutions which cannot be approximated in closed form; this is useful particularly when Bayesian and other non-parametric statistics are invoked. Some articles advocate the use of implied volatilities, and various other parameter calibration methods implied by option prices, in order to be consistent with market makers' decisions. Some articles concentrate on developing statistical methodology in parametric and non-parametric contexts, for discrete and continuous models. Others propose new investigative terrain, with uncertainty terms involving jumps, or long memory, in order to incorporate SV features which are difficult to describe using more classical models. Most methods are tested extensively using simulated or market data, and a number of topics have a definite statistical bend. These are signs that this Symposium's quantitative researchers are making sure their methods can be used to good effect by practitioners.

We now give a technical summary of the articles in this issue, which starts with studies of basic and extended SV models, then covers statistical aspects of volatility calibration and estimation, followed by studies of long-memory SV, and finally a treatment of portfolio optimization.

Stochastic volatility models have been advocated as a good modeling tool for option pricing because they are capable of explaining the volatility smiles, smirks, frowns, and other skews observed in the volatilities implied by option markets. However, not every SV model can be calibrated to explain every possible implied volatility skew. The Displaced Lognormal (DL) model is among the simplest and most popular SV models. It can be represented as merely a standard Black-Scholes model which is shifted in space by a constant value. It is chosen for its mathematical tractability and the fact that it exhibits a volatility skew. Yet Roger Lee and Dan Wang provide a mathematical proof that this model has some undesirable features, including a monotonicity constraint and a bounded slope on the implied volatility, which makes it poorly suited for computing equity options. However, they show that one should not discard this model even in the case of equity derivatives, since it can be used as a control variate in the Monte-Carlo computation of option prices in more flexible SV models, such as the Constant Elasticity of Variance (CEV), and especially its non-local generalization known as the SABR (stochastic alpha-beta-rho). They illustrate the efficiency of their 
technique by computing continuous-time barrier option prices under CEV and SABR, and by showing how to calibrate the DL model for this purpose, based on the level and slope of observed implied volatilities.

The capital asset pricing model in continuous time is a convenient way of expressing the dynamics of a specific asset as they correlate to those of the entire market, via the correlation coefficient $\beta$ (the beta, or "slope" coefficient). This model can also be endowed with a stochastic market volatility component, just as in a standard Hull-White model or any stochastic volatility framework, in order to account for implied volatility skews. In order to have a more realistic dependence of the asset on the market movements, it makes sense to allow the beta to depend on the market state itself: during tough overall economic conditions, trades will follow market movements more closely ("stressed beta"). Jean-Pierre Fouque and Adam Tashman propose increasing beta by a constant value $\delta$ when the market index falls below a predetermined threshold. Even when the underlying market volatilty is constant, this model results in stochastically switching asset volatility. Remarkably, in this model, a detailed probabilistic analysis reveals explicit expressions for option prices and replication. When the market volatility is stochastic, option pricing approximations can be obtained in the fast mean reverting regime. The stressed beta model's parsimony then allows for an efficient calibration of its parameters (market volatility, $\beta, \delta$, and the switching threshold $c$ ) against implied volatility and its affine relation to the logmoneyness-to-maturity ratio.

SV models are also capable of capturing the phenomenon by which volatility tends to increase when market prices decrease. This so-called leverage effect can be modeled by assuming that the noise term driving the volatility is correlated to the noise term driving the stock or market process; the leverage correlation coefficient would then typically be a negative constant, in order to describe the rise in volatility when bad news hits the market. In some cases, it may be preferable to allow several different leverage coefficients. Almut Veraart and Luitgard Veraart contend that volatility has some "stochastic volatility" of its own, and propose to model this with a stochastic leverage effect. More specifically they consider standard SV models, including the Heston and Barndorff-Nielsen-Shepard models, and use correlation coefficients which are driven by so-called Jacobi processes. Using linear transformations of these processes, one can specify a given sub-interval of $[-1,1]$ where the stochastic correlation will live (for instance, one can insist that correlation is always negative), and also ensure that it be mean-reverting at a specified speed. Option prices in these models are easily computed using straightforward Monte-Carlo simulations, because of the models' near explicit structure. The other parameters in the SV models can then be adjusted to account for a number of different volatility skew shapes; the authors explain how to produce specific symmetries, asymmetries, convexities, and shifts in implied volatilities. In their models, they also investigate the mathematical and statistical aspects of empirical leverage, empirical volatility feedback, and non-parametric realized variances and covariances.

The estimation of volatility based on high-frequency data is at the core of very general questions in stochastic calculus, such as the representation of quadratic variation, but it is also a topic of much current interest and empirical work in finance. In 
financial econometrics, making simplifying modeling assumptions is often considered as a better idea than trying to described many features of financial data by developing highly complex models. Keeping with this parsimonious philosophy, Per Mykland considers the so-called conditionally Gaussian SV framework, also referred to as the assumption of "no-leverage effect". Therein a multiple-asset model's volatility matrix (quadratic variations and covariations) may be random, but it is observable; given this volatility observation, the model's log returns are Gaussian, even if observed in asynchronous discrete time. A number of different statistical mathods are available in this situation, for which Mykland develops a theory based on an approximation technique he calls locally constant volatility. It allows one to replace the market dynamics by ones in which volatilities remain constant in predetermined small intervals, in which the model break points need not have any relation with the market observation times beyond some mild assumptions. The full volatility matrix of the approximate market, using all observation times, will typically have a number of unobserved values. Nevertheless, Mykland shows a contiguity theorem: parameter consistency under the approximate model implies consistency under the original model; the price to pay for the approximation is a bias in the asymptotic distribution. As applications of this theory, in which this bias is typically zero, Mykland proposes an ANOVA with multiple regression and finite smoothing, to estimate the residual quadratic variation of a given process after regressing on multiple others. He also shows how to construct a simple moments-based estimator of the variance in the maximum likelihood (a.k.a. Hayashi-Yoshida) estimator for the volatility matrix.

Realized volatility based on historical data, and implied volatility based on current option prices and the Black-Scholes formula, are two aspects of the same quantitative question. Practitioners generally regard implied volatility as containing more information than its realized counterpart, in the sense that it helps understand forecasting, which is not possible in a short-memory context using only historical data. Yet because the two concepts are tightly related, it is worth trying to explain this quantitatively. Lan Zhang does this in a general context, in which she shows in an SV framework that, in accordance with econometric intuition, realized volatility coincides with instantaneous implied volatility when the latter exists. However, she also shows that it normally does not exist, because cumulative implied volatility is typically diffusive. In this case, she derives no-arbitrage identities and inequalities, and shows how to use diffusive implied volatility to hedge away some of the Gamma and Vega risk. Hedging using implied volatility is desirable in order to minimize reliance on raw option prices whose dependence on moneyness is high. In order to deal with hedging in incomplete multifactor SV models, in which the goal is to minimize the hedging error, Zhang proposes two schemes for empirical model selection to choose an optimal number of hedging instruments. One method is to use F-testing, the other method works through cross-validation.

Since change-point detectors are statistically unreliable, and econometric misspecifications are often caused by structural breaks, it is important to identify which models are most robust to these kinds of misspecifications. Mihaela Craioveanu and Eric Hillebrand compare the behavior of various vector auto-regressive (VAR) models under the influence of unknown level shifts, when estimation ignores these shifts. It is known that the sum of the autoregressive coefficients approaches 1 for 
high-frequency data when the level shift is large; in practice this amounts to an implied average mean-reverting time which tends to infinity, or at least is much larger than the data's true mean-reversion parameter. Using theoretical analyses and simulations, the authors show that this effect is much more marked for estimators which do not include an external source of randomness in the volatility, such as GARCH's quasi maximum likelihood estimator, while for those that do, such as least squares for VAR models, the estimation bias is lessened for models where volatility of volatility is high in comparison with the level shifts, a trade-off which does not exist for GARCH models. The resulting much higher robustness of VAR models compared to GARCH models, is clearly illustrated in practice by estimating the mean reversion of stock data from the Dow Jones Industrial Average using $\operatorname{GARCH}(1,1)$, as opposed to using HAR-RV(1,5,21), a common VAR model with daily, weekly, and monthly lag scales.

Stochastic volatility models in continuous time do not need to be restricted to diffusion processes. In order to account for non-constant volatility structures in very short term options, there is beginning to be a consensus that jump-diffusion processes of Lévy type are perhaps the best modeling tool. Such processes exhibit jump activity with various jump-size intensities, controlled by the so-called Lévy measure, which gives as much non-parametric flexibility as any continuous SV model. A challenging issue is to estimate the intensity of small jumps; a popular power parametrization enables framing this question using the so-called (Blumenthal-Getoor) jump index. For financial applications, the essential feature of volatility clustering, which say that high levels of volatility tend to occur in concentrated periods, can be incorporated into jump-diffusion processes in a much more direct way than via continuous SV models: by using a random clock, one can directly see that trading activity levels correspond to high volatility levels. In his paper, José-Enrique Figueroa López proposes non-parametric techniques to statistically determine the jump index, and to track the random clock using a threshold, based on high-frequency observations. He establishes strongly consistent estimators, presents their performance on simulated data, and uses them successfully on Intel stock data, showing evidence of the importance of using very high-frequency data to have a good-quality estimator of the jump activity index, which in turn allows a clear tracking of the random clock, i.e. a dynamic identification of the periods of high volatility, and finally a non-parametric estimation of integrals of the Lévy measure for large jumps.

In the term structure of implied volatility in which long memory has been consistently reported, the persistence of implied volatility patterns even in long-term options is difficult to explain by short term memory assumptions on the volatility. Moving beyond the standard continuous-time models (Hull-White, Heston, etc...), Fabienne Comte, Laure Coutin, and Eric Renault take up a strategy that was defined by Comte and Renault in 1998, in which long memory is introduced in a continuous-time stochastic volatility model by integrating a stationary ergodic process fractionally. In the 1998 article, the underlying model was an exponentiated Ornstein-Uhlenbeck process. In this Symposium, the authors argue that moving away from such a log-normal base is warranted. They propose using an affine extension of the square-root process. Its fractional integration has the following feature which makes it particularly suitable in option pricing: the volatility's long memory is visible only via its expectations, not 
its higher moments. It implies that in practice, one only needs to keep track of one long memory stochastic process, such as at-the-money implied volatility, while the remainder of the relevant pricing scheme can follow standard continuous-time Markovian practices. Because the long memory square root process has a small number of tuning parameters with simple financial interpretations, their estimation from high-frequency data, combining a method of moments and fractional calculus, are reasonably straightforward. The article of Comte, Coutin, and Renault also has an important theoretical component: beyond the mathematical dexterity required to prove their theorems, they show that there are explicit formulas for all moments of all integrated volatilities (integrals of the squared volatility process over any time interval). These formulas can then be used to analyze the long-memory effects in some exact quantitative detail. One important conclusion is that long-memory models are much more effective at explaining smiles in long-term options than short memory models, while the opposite is true for very short term options. This helps elucidate the modeling difficulties which are encountered in trying to fit short-memory SV models to short-term and long-term options simultaneously. The authors' formulas are wieldy enough that they can compute the spectral density of the entire integrated volatility process, showing in particular that it shares the same level of long memory as the spot volatility process, and as the expected integrated volatility process.

The archetypical long-memory stochastic process is the so-called fractional Brownian motion (fBm). The fBm has the advantage of being an explicit Gaussian process which generalizes standard Brownian motion in many directions, including the stationarity and self-similarity of its increments. Similarly to the model class of Comte, Coutin and Renault, fBm can be represented using a fractional integral representation of a kernel integrated against standard Brownian motion, but the formalism of fractional integral calculus is often unnecessary with $\mathrm{fBm}$. An explicit mean-reverting ergodic process is obtained as the generalized Ornstein-Uhlenbeck process driven by fBm. Alexandra Chronopoulou and Frederi Viens propose to use this kind of explicit process as a basis for a SV model with long-memory. They develop a complete numerical scheme based on discrete observations for the purpose of option pricing. In order to track the volatility process, they use a Bayesian interacting particle technique known as $S V$ filtering. A multinomial recombining tree is constructed to price options, based on the empirical filtered volatility distribution. In principle this non-parametric technique can work for any SV model for which the SV filter converges as the number of particles increases. It has the advantage of containing any uncertainty on the volatility as empirically built-in. For the estimation of the underlying SV model, Chronopoulou and Viens show that the key question is to determine the long-memory parameter $H$ (the so-called Hurst parameter), while volatility filtering and multinomially-computed option prices are rather insensitive to other SV parameters. Because a consistent estimation of $H$ based on discrete stock data is not available, they choose instead an implied method, where $H$ is calibrated to liquid option prices. An analysis of S\&P 500 data shows that the option pricing scheme under long memory is particularly appropriate within a few months of market shocks.

The last topic investigated in this special issue is the question of portfolio optimization under stochastic volatility. As a generalization of classical Merton optimization 
via stochastic control, it has attracted less interest in recent years than the analysis of volatility, via and for the purpose of option prices. From a mathematical standpoint, this can be blamed on the fact that optimization, a non-linear problem, is more difficult to solve numerically and generally less robust, than pricing, which is linear. Nevertheless, any market with an implied volatility skew indicates an SV situation; then, if an SV model has been selected, for instance, by way of calibration against option prices, any procedure for portfolio selection in the same market must be consistent with SV. Ha-Young Kim and Frederi Viens approach this problem in the Merton context, by working in a standard continuous-time SV framework, and solving the control problem on the sole basis of discrete-time stock observations, via a Bellman scheme which is evaluated by Monte-Carlo simulation. This implies that an estimate of the SV filter is needed, and they choose the same interacting particle method mentioned above. The authors' motivation is to include discrete transaction costs. They show that even in the case of proportional costs, there is a minimal trading frequency below which it is suboptimal to engage in trading. Their analysis can be interpreted in the case of fixed transaction costs, as in the case of day traders, where the minimal trading frequency also appears as a maximal frequency, i.e. trading too often will also result in a suboptimal portfolio.

The articles in this Symposium are at the forefront of mathematical and statistical methods for stochastic volatility. They open a number of new directions of research, which we hope will be taken up by other researchers interested in quantitative and computational finance, and by those who seek new frameworks for understanding uncertainty in financial data.

We thank the Editor-in-Chief of the Annals of Finance, Professor Anne Villamil, for making the edition of this important Symposium possible, and all its contributors for sharing some of their best original research, and for working tirelessly towards perfecting their manuscripts. Above all, we thank the late Professor Charalambos (Roko) Aliprantis, who initiated this special issue as Founding Editor of the Annals of Finance. Thank you, Roko, for all you have done.

Frederi G. Viens, West Lafayette, Indiana, USA, June 30, 2010. 